\title{
ANALYSIS OF HEAT AND MASS TRANSFER ON STEADY MHD CASSON FLUID FLOW PAST AN INCLINED POROUS STRETCHING SHEET WITH VISCOUS DISSIPATION AND THERMAL RADIATION
}

\author{
V. MANJULA ${ }^{1} \&$ K. V. CHANDRA SEKHAR ${ }^{2}$ \\ ${ }^{I}$ Research Scholar, Department of Mathematics, Koneru Lakshmaiah Education Foundation, Vaddeswaram, \\ Andhra Pradesh, India \\ ${ }^{2}$ Associate Professor, Department of Mathematics, Koneru Lakshmaiah Education Foundation, Vaddeswaram,
} Andhra Pradesh, India

\begin{abstract}
The current paper examines the problem of steady MHD, natural convection flow of a Casson fluid past a slanted porous sheet under the influence of viscous dissipation and radiation. Non-linear governing equations are transformed into ordinary differential equations using transforms of equality. The obtained equations are interpreted using the fourthorder Runge Kutta method with the shooting method. The effects of various physical parameters on speed, temperature and concentration are depicted graphically.

KEYWORDS: Angle of Inclination, Magnetic Field, Eckert Number, Radiation Parameter, Porosity \& Chemical Reaction Parameter
\end{abstract}

Received: Mar 20, 2020; Accepted: Apr 10, 2020; Published: May 04, 2020; Paper Id.: IJMPERDJUN202027

\section{NOMENCLATURE}

Electrical conductivity

$\sigma_{1} \quad$ Stefan-Boltzmann constant

$\rho \quad$ Density of the fluid

$\psi \quad$ Stream function

$\theta \quad$ Dimensionless temperature

$T_{m} \quad$ Free stream temperature

$T_{w} \quad$ Uniform sheet temperature

K Thermal conductivity

$q r \quad$ Heat flux

C Concentration 


$\begin{array}{ll}C_{m} & \text { Free stream concentration } \\ \varphi & \text { Dimensionless concentration } \\ C_{w} & \text { Concentration of the sheet at the wall } \\ \mathrm{K} & \text { Thermal conductivity } \\ \mathrm{K}_{1} & \text { Mean absorption coefficient } \\ \mathrm{K} & \text { Porosity }\end{array}$

\section{INTRODUCTION}

In recent years, progress has been made in the study of the interaction of radiation with natural convection, due to its applications in many technological methods such as paper generation, crystallization and aerodynamics. The study plays a vital role in the temperature transferal analysis in the industry. Ostrach [1] focused on the free convection flow and temperature transferal of the fluid with uniform temperature. Hossain et al. [2] examined the radiation effects on natural convection flow of a gas over an infinite flat plate. Magyari and Keller [3] focused on the temperature transferal and mass transfer of an incompressible viscous fluid from an exponentially stretching vertical surface. Chen et al. [4] explored the study of temperature and mass transferal by Magneto Hydro Dynamic flow from a Permeable, inclined Surface with the effect of changeable Wall Temperature. Anjali Devi [5] discussed the effect of Viscous Dissipation on non-linear MHD flow over a stretching surface in a Pervious Media. Ishak [6] presents the MHD flow over an elongated sheet with the impact radiation. Besides, Firdous et al. [7] describes the effects of thermophores and synthetic responses on MHD in rotating liquid. Pavitra and Girisha [8] examined the flow of barrier layers in surfaces under the influence of viscous dissipation, internal heat production and consumption. Nadeem et al. [9] investigated the oblique movement of Casson fluids with rotation at steady temperature levels. Mukhopadhyay [10] provided a clear indication of the flow of Casson fluids under thermic fallout, suction or injection. Mathematical analysis of Magnetohydrodynamic flow of Casson fluid comprises thermic fallout handled by Shateyi and Marwo [11]. Mahanta et al. [12] performed a 3D Casson fluid flow over a previous elongated sheet. The results of slip on the magnetohydrodynamic stream of Casson fluid on the elongate permeable surface beneath the influence of heat sources, heat radiation, and synthetic responses have been analyzed by Saidulu et al. [13]. Next, K. Bhattacharya [14] provided valuable data on the concept of the flow of a Casson liquid across a surface with a temperature transferal impact. According to Pushpalatha et al. [5] fluid the subject of time-dependent in a Casson fluid flow constrained by a smooth plate changing in a rotational configuration. M. EswaraRao and S. Sreenadh [16] gave meaningful contributions towards the MHD Flow of Casson liquid across an elongated and Shrinking Sheet into Pervious Media. Many researchers have examined the outcomes of radiation. Pramanik [17] investigates the flow of Casson liquid at the ubiquity of thermal diffusion on the pore-expanding surface. R. Srinivasa Raju et al. [18] acknowledged the free convective MagnetoHydroDynamic flow of Casson fluid on the upward surface. They studied the effects of the chemical reaction, the angle of the slope, the viscous dispersion and the constant heat flow. Furthermore, Hasan [19] et al. have implemented extensive research into Casson fluid with the effects of temperature transferal on the expanding facade. The present analysis focuses on detecting the effects of temperature and mass transferal on the flow of MHD Casson fluid behind an inclined porous expanding sheet under the influence of radiation. The governing equations are resolved using the shooting technique with the fourth-order RK method. 


\section{MATHEMATICAL MODEL}

Consider a continuous two-dimensional flow of a chemically responsive fluid. It is an impenetrable, conductive viscous fluid along a permeable stretch sheet. It is tilted vertically by alpha planted in a pervious media with energy production or consumption. Apply a strong magnetic field along the y-axis. Here, unlike the applied magnetic range, the effect of the induced magnetic range is sufficiently weak. When the fluid is an electrical insulator, the current flowing through the fluid creates an induced magnetic range. Since the fluid is conductive, there is only an applied magnetic range of force B0 that exerts a magnetic force in the $\mathrm{x}$-axis. Introduce a pair of equal and opposite forces towards $\mathrm{x}$-direction. Then the sheet is expanded. It is shown in the figure.

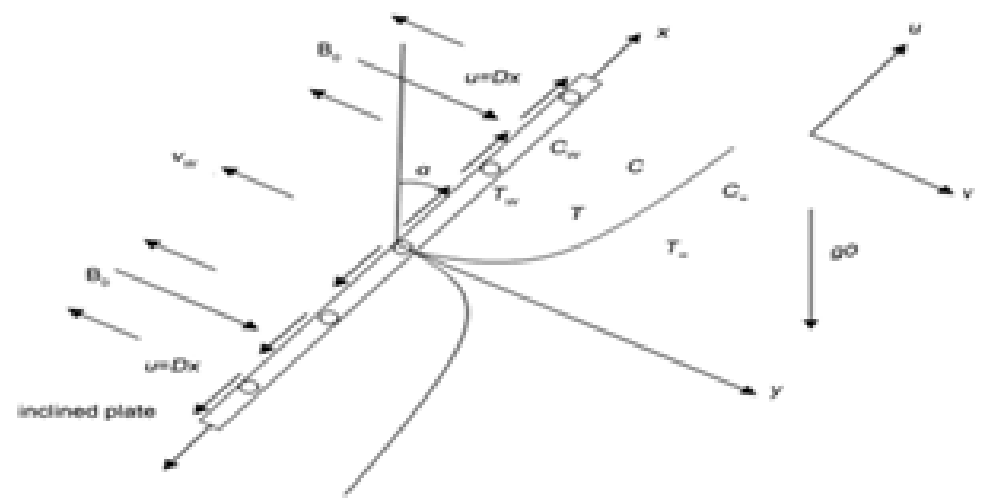

Geometry of the Problem.

Using Boussinesq approximation the equations are

$$
\begin{aligned}
& \frac{\partial u}{\partial x}+\frac{\partial v}{\partial y}=0 \\
& u \frac{\partial u}{\partial x}+v \frac{\partial u}{\partial y}=v\left(1+\frac{1}{\beta}\right) \frac{\partial^{2} u}{\partial y^{2}}+g \beta_{T}\left(T-T_{\infty}\right) \cos \alpha+-\frac{\sigma B_{0}^{2} u}{\rho}-\frac{v u}{k}-\frac{b u^{2}}{k} \\
& u \frac{\partial T}{\partial x}+v \frac{\partial T}{\partial y}=\frac{k}{\rho c_{p}} \frac{\partial^{2} T}{\partial y^{2}}+\frac{Q_{0}}{\rho c_{p}}\left(T-T_{\infty}\right)-\frac{1}{\rho c_{p}} \frac{\partial q_{r}}{\partial y}+\frac{v}{c_{p}}\left(1+\frac{1}{\beta}\right)\left(\frac{\partial u}{\partial y}\right)^{2} \\
& u \frac{\partial C}{\partial x}+v \frac{\partial C}{\partial y}=D_{m} \frac{\partial^{2} C}{\partial y^{2}}-K_{r}^{2}\left(C-C_{\infty}\right)
\end{aligned}
$$

The corresponding boundary conditions are at $\mathrm{y}=0$

$$
\begin{aligned}
& u=D x, v=v_{w}(x), T=T_{w}, C=C_{w} \\
& u=0, T=T_{\infty}, C=C_{\infty} \quad \text { as } \mathrm{y} \rightarrow \infty
\end{aligned}
$$

By the Roseland approximation for Radiation 


$$
\frac{\partial q_{r}}{\partial y}=\frac{-4 \sigma}{3 k} \frac{\partial T^{4}}{\partial y}
$$

The temperature difference within the flow is a linear function of temperature

$$
T^{4} \cong 4 T_{\infty}^{3} T-T_{\infty}^{4}
$$

Using (7) in (3) gives

$$
u \frac{\partial T}{\partial x}+v \frac{\partial T}{\partial y}=\frac{k}{\rho c_{p}} \frac{\partial^{2} T}{\partial y^{2}}+\frac{Q_{0}}{\rho c_{p}}\left(T-T_{\infty}\right)+\frac{v}{c_{p}}\left(1+\frac{1}{\beta}\right)\left(\frac{\partial u}{\partial y}\right)^{2}+\frac{16 \sigma_{1} T_{\infty}{ }^{3}}{3 \rho c_{p} k_{1}} \frac{\partial^{2} T}{\partial y^{2}}
$$

\section{SIMILARITY ANALYSIS}

The following are the similarity transformations used to find the solution.

$$
\eta=y \sqrt{\frac{D}{v \operatorname{Re}_{x}}}, \psi=x \sqrt{\frac{v \operatorname{Re}_{x}}{D}} f(\eta), \theta(\eta)=\frac{T-T_{\infty}}{T_{w}-T_{\infty}}, \phi(\eta)=\frac{\mathrm{C}-C_{\infty}}{\mathrm{C}_{w}-C_{\infty}}
$$

Using the above transformations equations (2), (8) and (4) takes the form.

$$
\begin{aligned}
& \left(1+\frac{1}{\beta}\right) f^{\prime \prime \prime}(\eta)+\frac{\mathrm{Re}_{x}}{D} f(\eta) f^{\prime \prime}(\eta)-\left(M+\frac{k}{D a}\right) f(\eta)+-\left(M+\frac{F(s)}{D a}\right) \frac{\mathrm{Re}_{x}}{D} f^{\prime}(\eta)^{2}-\gamma \theta \operatorname{Cos} \alpha \mathrm{Re}_{x}=0 \\
& \theta^{\prime \prime}(\eta)+\mathrm{P}_{n} \mathrm{R}_{e x} Q \theta+\left(\frac{\mathrm{P}_{n} E c}{D^{2}}\right) f^{\prime \prime}(\eta)^{2}+\frac{\mathrm{P}_{n} \mathrm{R}_{e x}}{D} \theta^{\prime}(\eta) f(\eta)=0 \\
& \phi^{\prime \prime}(\eta)+\frac{S c R_{e x}}{D}\left(f(\eta) \phi^{\prime}(\eta)-K r^{2} \phi(\eta)\right)=0
\end{aligned}
$$

where

$$
\begin{aligned}
& M=\frac{\sigma B_{0}^{2}}{\rho D}, D a=\frac{k}{x^{2}} \operatorname{Pr}=\frac{v \rho c_{p}}{k}, \quad N=\frac{k k_{1}}{4 \sigma_{1} T_{\infty}^{3}}, \quad S_{c}=\frac{v}{D_{M}} \\
& \operatorname{Re}_{x}=\frac{x^{2} D}{v} \quad \mathrm{E}_{C}=\frac{D^{2} x^{2}}{C_{p}\left(T-T_{\infty}\right)}, \quad \gamma=\frac{\mathrm{Gr}_{x}}{\operatorname{Re}_{x}^{2}}, F_{S}=\frac{b}{x}, P_{n}=\frac{3 N P_{r}}{4+3 N}, Q=\frac{Q_{0}}{\rho C_{p} D}
\end{aligned}
$$

The corresponding boundary conditions are

$$
\begin{aligned}
& \mathrm{f}(\eta)=\mathrm{Fw}, \mathrm{f}^{\prime}(\eta)=1, \theta(\eta)=1, \varphi(\eta)=1 \text { at } \eta=0 \\
& \mathrm{f}(\eta)=0, \theta(\eta)=0, \varphi(\eta)=0 \text { as } \eta \rightarrow \alpha \text { where } \mathrm{Fw}_{\mathrm{w}}=\frac{\mathrm{v}_{\mathrm{w}}}{\sqrt{D U}}
\end{aligned}
$$

\section{NUMERICAL COMPUTATION}

The non-linear ode (10), (11) and (12), as well as the boundary conditions (14), have been extracted using fourth-order R $\mathrm{k}$ method by the Matlab code. 


\section{RESULTS AND DISCUSSIONS}

The velocity profiles corresponding to Casson parameter $\beta$, Magnetic range parameter (M), permeability parameter (K), Radiation parameter $(\mathrm{N})$, Reynolds number $(\mathrm{Re})$, suction parameter $(\mathrm{Fw})$, tilt angle $(\alpha)$ are described through numerical computation. The temperature difference related to the tilt angle, are Eckert number (EC), radiation parameter (N), Prandtl number Pr, heat source parameter (Q), Smidt number (SC). The basic parameters (Q), Eckert number (EC), and chemical reaction parameters $(\mathrm{kr})$ are solutions of the velocity, temperature, and concentration profiles, as shown in Figures 1-15. The speed attributes for several values $\alpha, \mathrm{k}, \mathrm{Re}, \mathrm{M}$, and Fw are shown in Figures 1-5. Figure 1 shows that increasing Casson parameters slows down the velocity. An increase in the Casson parameter is a decrease in liquid flow. Figure 2 exhibits the effect of magnetic parameters on velocity. The observations are that the magnetic force counteracts the flow of the liquid and hence the velocity of the liquid slows down as the magnetic parameter progresses. Figure- 3 shows that increasing permeability reduces velocity. Figure 4 shows that increasing the radiation parameter leads to increasing velocity due to decreasing buoyancy force. Similarly, the speed limit due to the addition of Reynolds number observed in Graph 5. Figure 6 shows the effect of the suction parameter on speed. As the constant suction stabilizes the expansion of the boundary layer, which reduces the momentum profile until it takes on a uniform shape. Figure 7 shows the result of the angle of inclination versus fluid motion. The speed decreases with increasing angle of inclination. Figure 8 shows the effect of Darcy number toward temperature. As the Darcy number Da increases, the permeability of the medium increases, increasing the reliability of the convection mode. Therefore, the temperature gradient increases with the addition of the Darcy number. According to the figure- 9 the temperature increases with the addition of the Eckert number. Figure 10 removes the effect of radiation on pigments at body temperature. Radiation improves with rising temperature. Figure 11 displays the effect of Prandtl number on speed. As a result, the thickness of the concentration boundary layer decreases. Graph 12 shows that as the thickness of the layer of concentration range drops, the concentration diminishes with higher Darcy number. Figure 11 shows the temperature change with the Prandtl number. As the Prandtl number reduces the thickness of the thermal boundary layer, it reduces the temperature. Figure 12 shows the effect of a heat source on temperature. The temperature rises due to the effect of the heat source on generating heat from the surface. Figure 13 shows the influence of the Schmidt number on the concentration. The drop in concentration leads to an increase in the Schmidt number. Figure-14 shows that the effect of concentration decreases with higher Darcy number, and results in decline in the thickness of the concentration boundary layer. According to Figure-15, the rise in the suction parameter shows the fall in concentration.

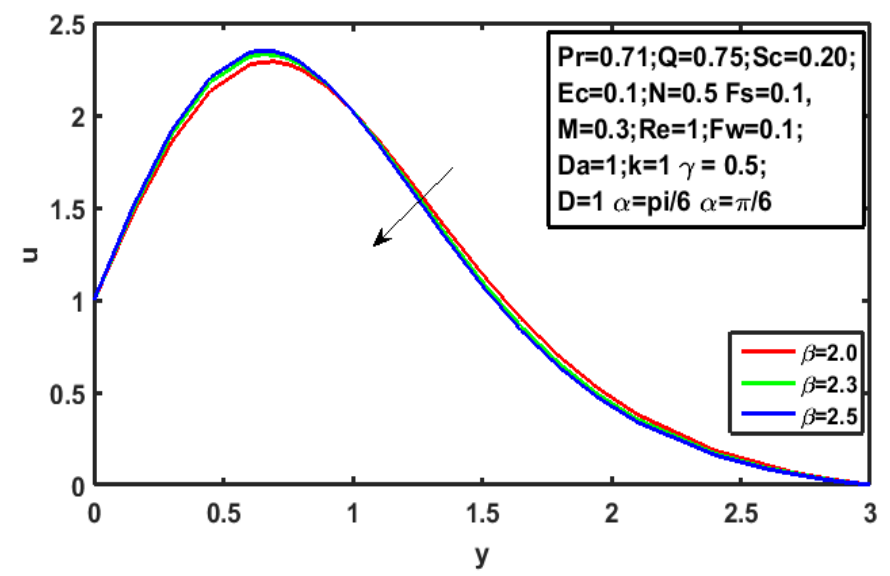

Figure 1: Effect of $\beta$ on Velocity. 


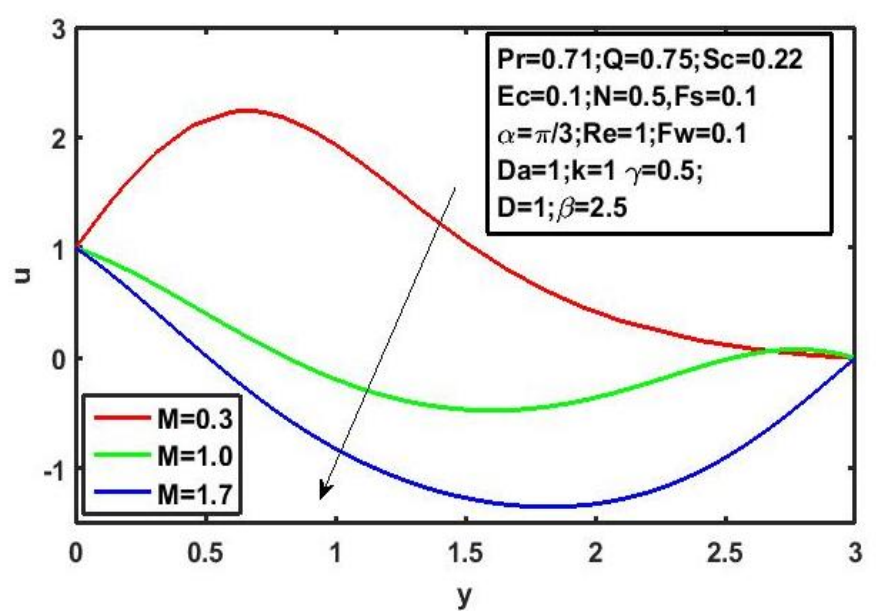

Figure 2: Impact of $M$ on Velocity.

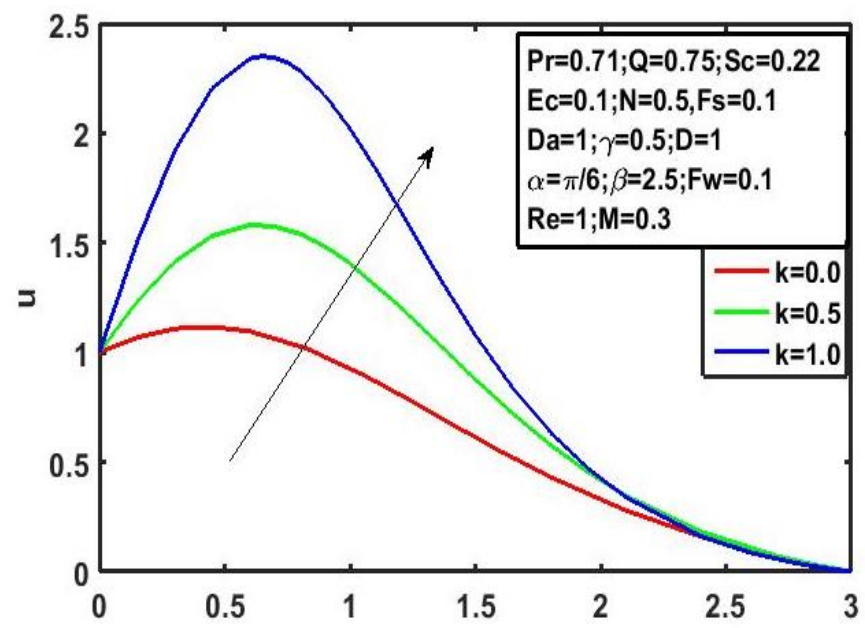

Figure 3: Influence of k on Velocity.

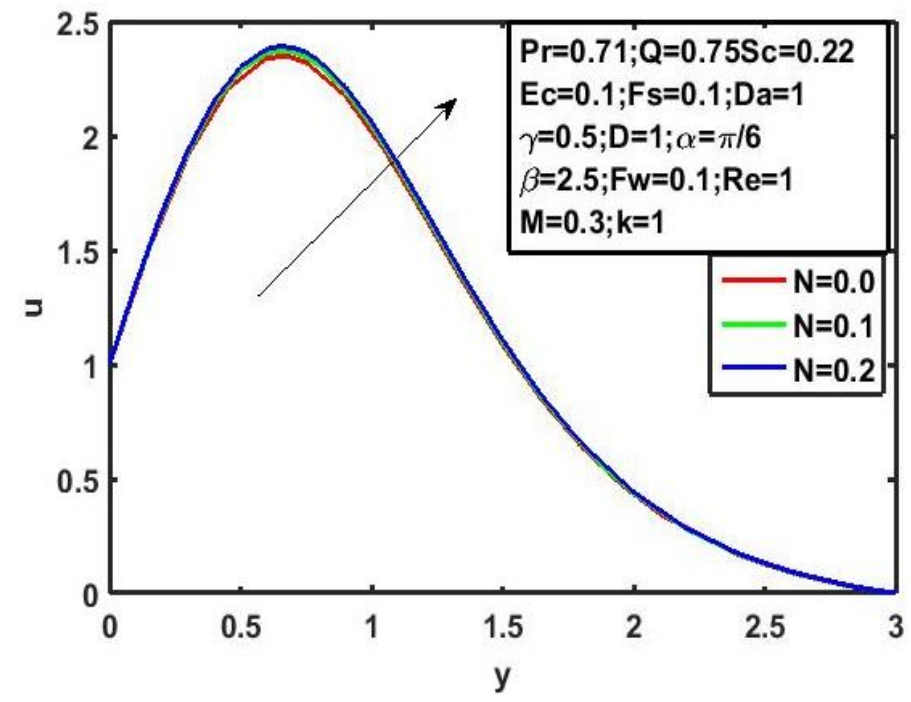

Figure 4: Variation of $\mathrm{N}$ on Velocity. 


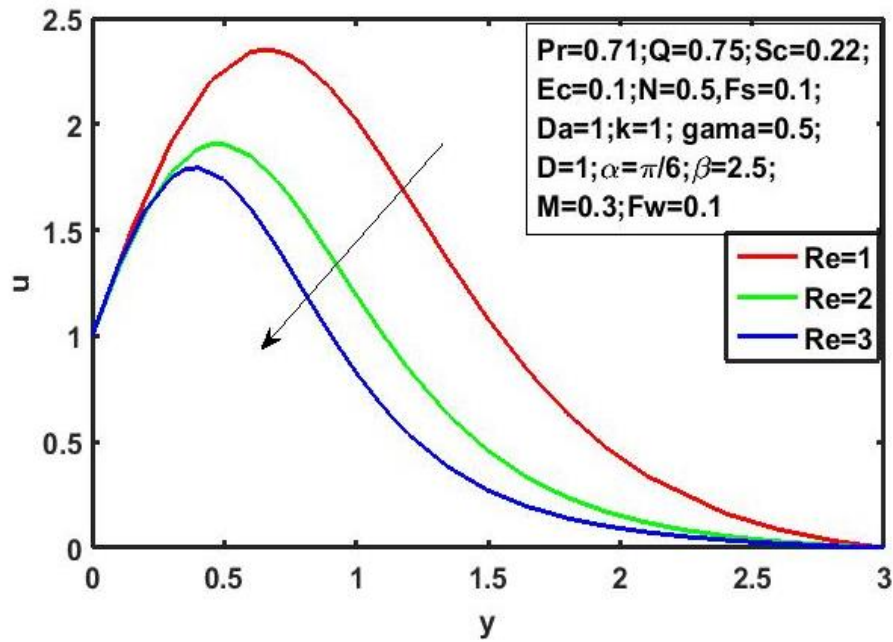

Figure 5: Effect of Re on Velocity.

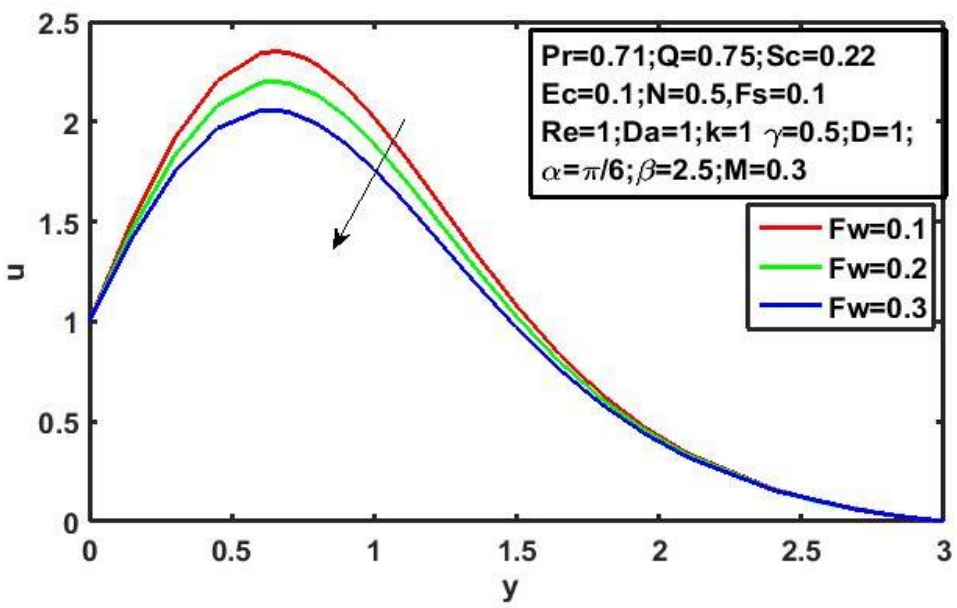

Figure 6: Effect of Fw on Velocity.

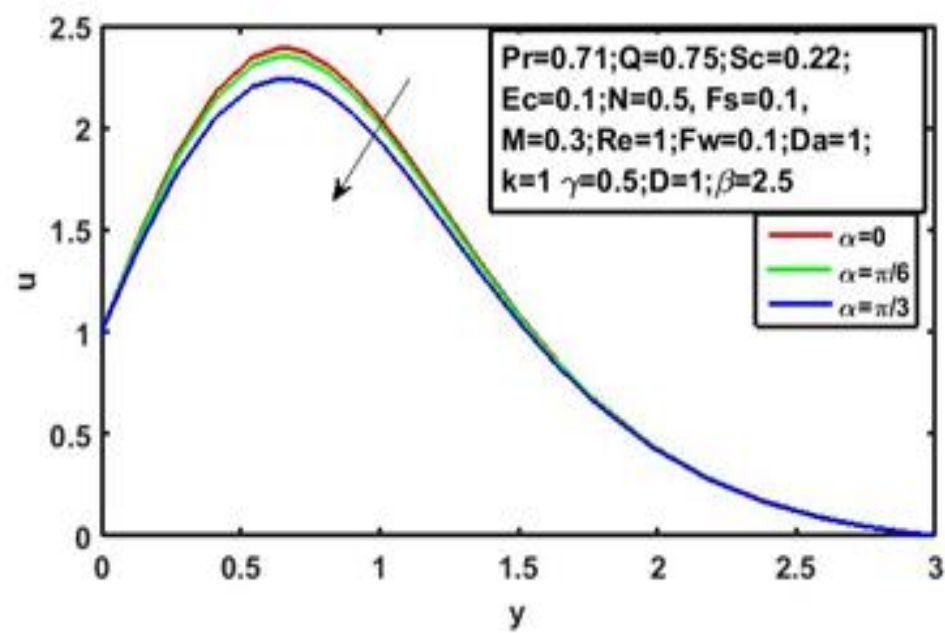

Figure 7: Impact of $\alpha$ on Velocity. 


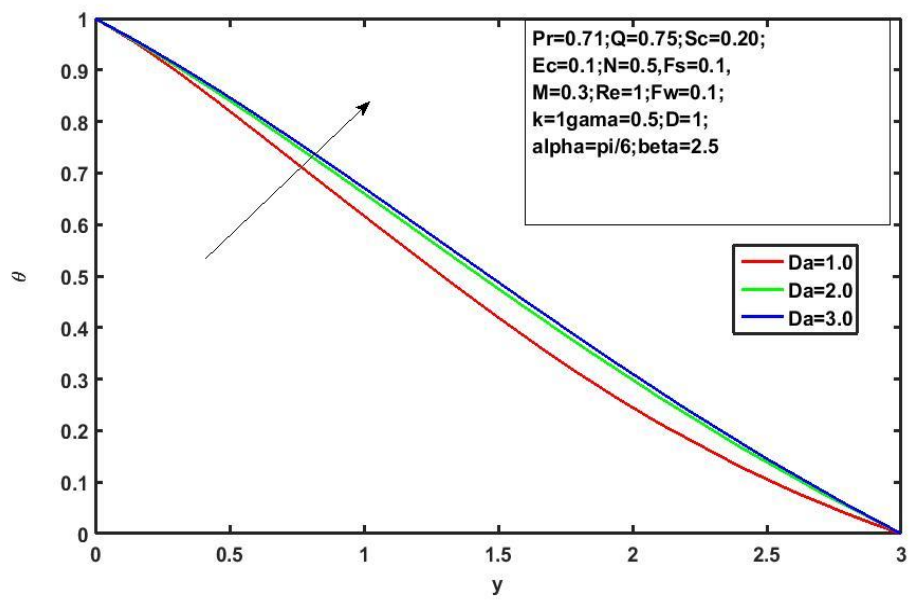

Figure 8: Influence of Da on Temperature.

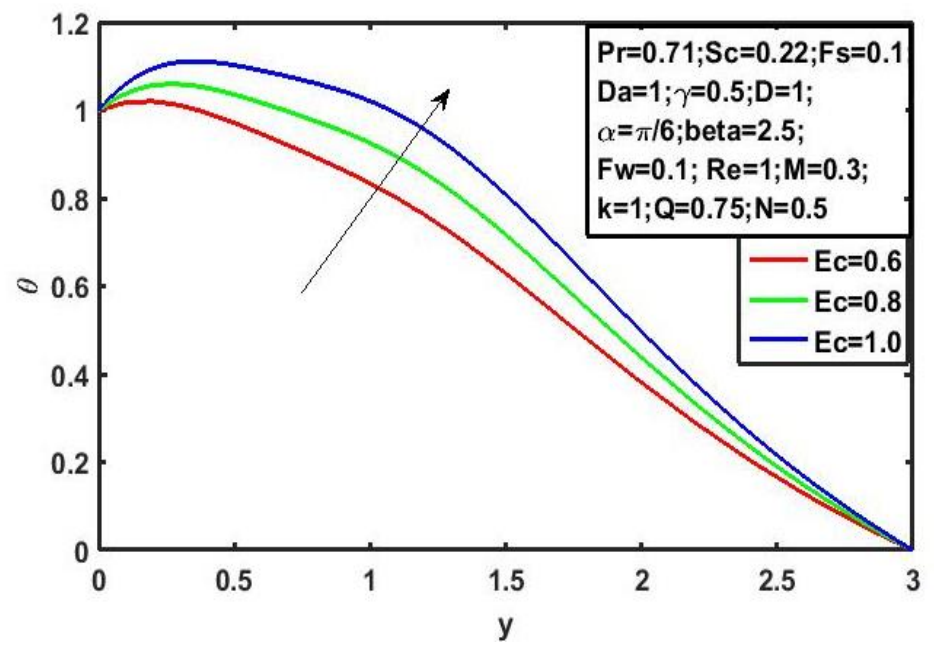

Figure 9: Effect of Ec on Temperature.

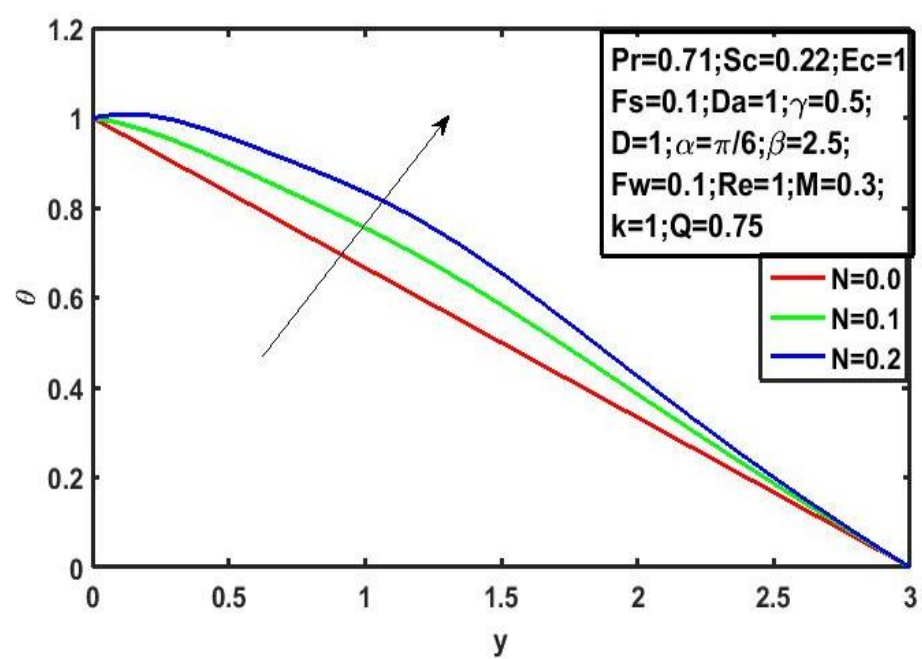

Figure 10: Influence of $\mathrm{N}$ on Temperature. 


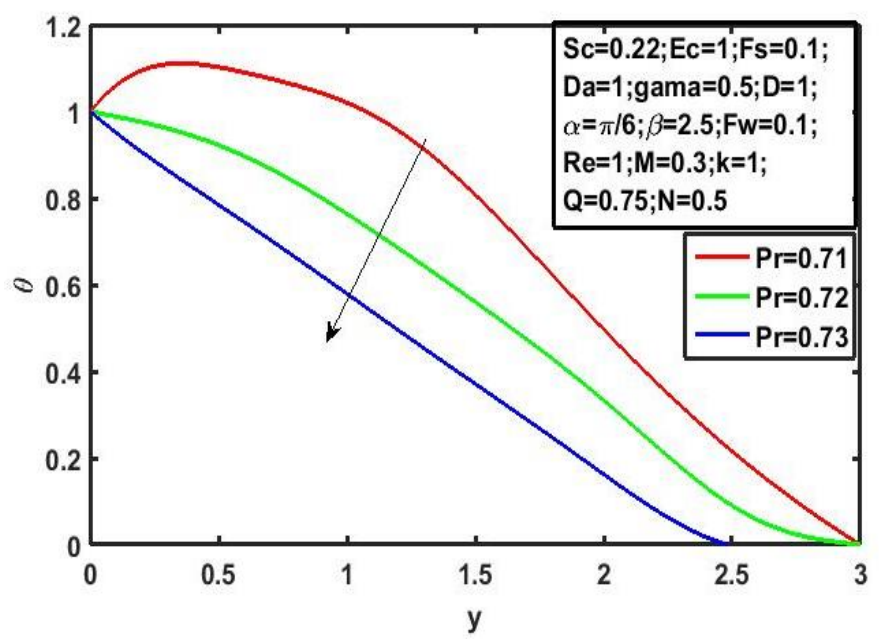

Figure 11: Variation of Pr on Temperature.

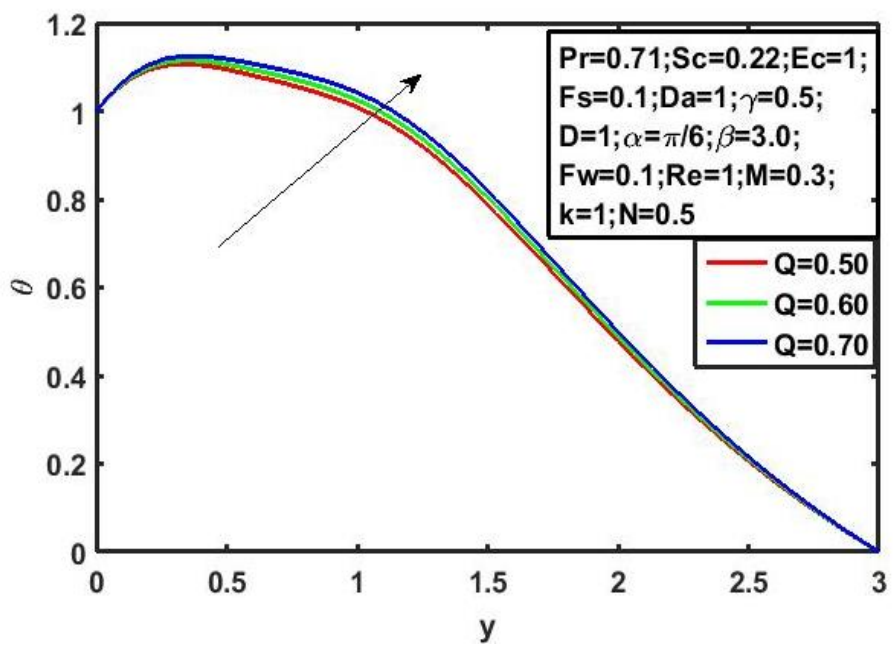

Figure 12: Impact of $Q$ on Temperature.

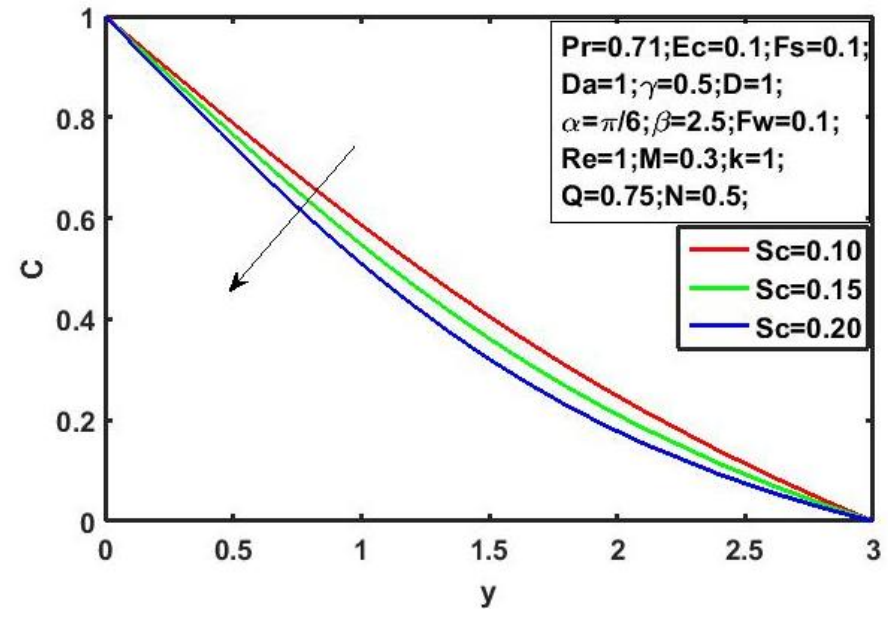

Figure 13: Influence of Sc on Concentration. 


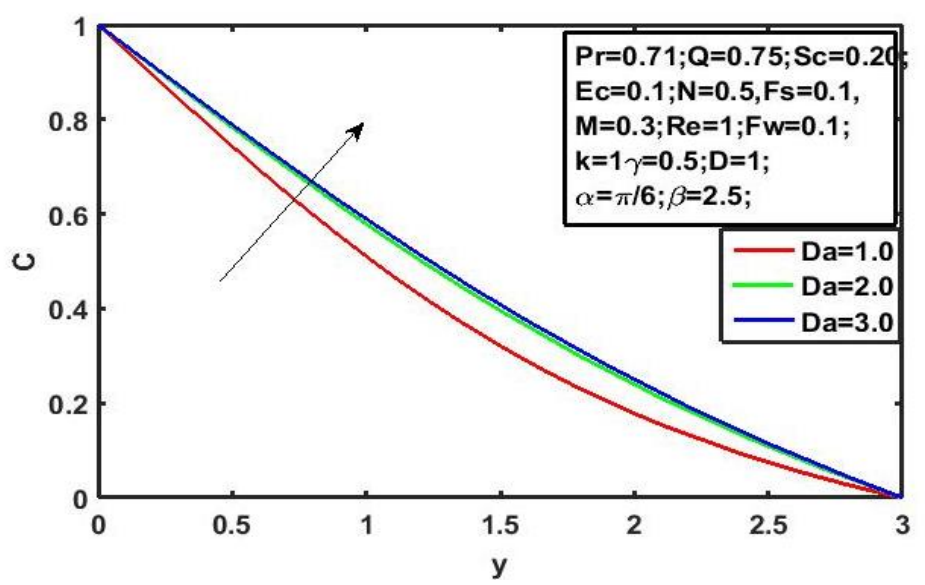

Figure 14: Impact of Da on Concentration.

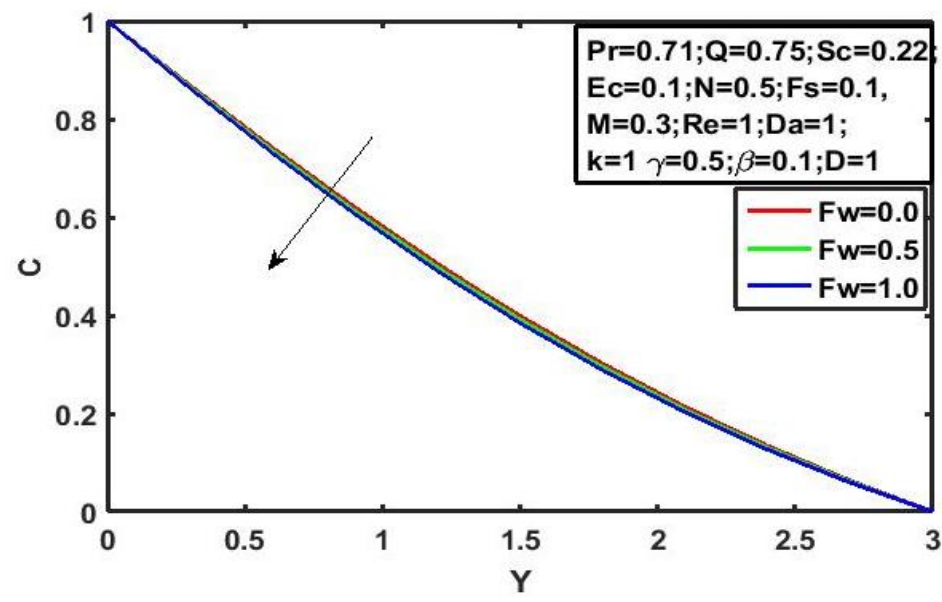

Figure 15: Effect of Fw on Concentration.

\section{CONCLUSIONS}

The present analysis influence different physical parameters that are covering the MHD free convective flow past inclined sheet. In similar, transformations used by Hasan et al. [7] for velocity segments along the x-direction and along y-direction are $\mathrm{u}=\operatorname{Dxf}(\eta), \mathrm{v}=-\sqrt{D V} \mathrm{f}(\eta)$. However, in the present discussion, the transformations given by equation contain the Reynolds number. For this reason, we have shown the contribution of the Reynolds number to the speed, temperature, and concentration profiles. However, the conclusions agree with Hassan et al. [7]. The primary purpose of this edition is to use it widely in the industry to determine the type of final product. In the end, the results are as follows:

- Velocity reduces with a rise in Casson parameter, magnetic parameter, Reynolds number and suction parameter.

- With rise in velocity, the magnitude of permeability parameter $\mathrm{K}$ and radiation parameter $\mathrm{N}$ increases.

- With the rise in temperature, the Ekret number Ec, the radiation parameter $\mathrm{N}$, the heat source $\mathrm{Q}$ and Darcy number Da increases.

- The temperature declines with rise in Prandtl number Pr.

- The concentration declines with the rise in the Schmidt number and suction parameter. 
Finally, the effects of skin - friction, rate of heat and mass transfer coefficients are displayed in the table-1.

Table 1

\begin{tabular}{|c|c|c|c|c|c|c|c|c|c|c|c|}
\hline Da & $\boldsymbol{\beta}$ & $\mathbf{P r}$ & $\mathbf{Q}$ & $\mathbf{E c}$ & $\mathbf{N}$ & $\boldsymbol{k}$ & $\boldsymbol{R e}$ & $\boldsymbol{F} \boldsymbol{w}$ & $\boldsymbol{f}(\mathbf{0}$ & $-\boldsymbol{\theta}(\mathbf{0})$ & $-\boldsymbol{\varphi}(\mathbf{0})$ \\
\hline 1 & 2 & 0.71 & 0.75 & 0.1 & 0.5 & 1 & 1 & 0.1 & 4.9464 & 0.2961 & 0.5198 \\
\hline 1 & 2.2 & 0.71 & 0.75 & 0.1 & 0.5 & 1 & 1 & 0.1 & 4.9629 & 0.2922 & 0.5210 \\
\hline 1 & 2.5 & 0.71 & 0.75 & 0.1 & 0.5 & 1 & 1 & 0.1 & 4.9734 & 0.2872 & 0.5222 \\
\hline 1 & 2.5 & 0.71 & 0.75 & 1 & 0 & 1 & 1 & 0.1 & 4.9677 & 0.3333 & 0.5223 \\
\hline 1 & 2.5 & 0.71 & 0.75 & 1 & 0.05 & 1 & 1 & 0.1 & 0.9905 & 0.2053 & 0.5230 \\
\hline 1 & 2.5 & 0.71 & 0.75 & 1 & 0.1 & 1 & 1 & 0.1 & 5.0125 & 0.0826 & 0.5237 \\
\hline 1 & 2.5 & 0.71 & 0.75 & 0.2 & 0.2 & 1 & 1 & 0.1 & 4.9929 & 0.1831 & 0.5419 \\
\hline 1 & 2.5 & 0.71 & 0.75 & 0.2 & 0.3 & 1 & 1 & 0.1 & 5.0126 & 0.0770 & 0.5426 \\
\hline 1 & 2.5 & 0.75 & 0.75 & 0.2 & 0.5 & 1 & 1 & 0.1 & 0.6927 & 1.0199 & 0.4650 \\
\hline 1 & 2.5 & 0.1 & 0.75 & 0.2 & 0.5 & 1 & 1 & 0.1 & 4.9918 & 0.1971 & 0.5421 \\
\hline 1 & 2.5 & 0.71 & 0.75 & 0.2 & 0.5 & 0.5 & 1 & 0.1 & 2.3748 & 0.0594 & 0.4910 \\
\hline 1 & 2.5 & 0.71 & 0.75 & 0.2 & 0.5 & 1.5 & 1 & 0.1 & 3.8254 & 1.7559 & 0.5714 \\
\hline 1 & 2.5 & 0.71 & 0.75 & 0.2 & 0.5 & 1.5 & 1 & 0.9 & 2.3045 & 0.1942 & 0.5840 \\
\hline 1 & 2.5 & 0.71 & 0.75 & 0.2 & 0.5 & 1.5 & 1 & 1.5 & 0.8217 & 0.3740 & 0.6367 \\
\hline 1 & 2.5 & 0.71 & 0.75 & 0.2 & 0.5 & 1.5 & 0.1 & 0.1 & 2.7016 & -1.1107 & 0.3541 \\
\hline 1 & 2.5 & 0.71 & 0.75 & 1 & 0.5 & 1 & 0.2 & 0.1 & 4.8581 & -1.0460 & 0.3890 \\
\hline 1 & 2.5 & 0.71 & 0.75 & 1 & 0.5 & 1 & 0.3 & 0.1 & 6.3032 & -1.8457 & 0.4263 \\
\hline 1 & 2.5 & 0.71 & 1 & 1 & 0.5 & 1 & 1 & 0.1 & 0.1833 & 0.7311 & 0.4416 \\
\hline 1 & 2.5 & 0.71 & 1.5 & 1 & 0.5 & 1 & 1 & 0.1 & 5.2207 & -0.9943 & 0.5496 \\
\hline 1.5 & 2.5 & 0.71 & 0.75 & 1 & 0.5 & 1 & 1 & 0.1 & 1.5388 & 0.1734 & 0.4695 \\
\hline 2 & 2.5 & 0.71 & 0.75 & 1 & 0.5 & 1 & 1 & 0.1 & 0.7845 & 0.2254 & 0.4498 \\
\hline
\end{tabular}

\section{REFERENCES}

1. Ostrach, S. Laminar Natural Convection Flow and Heat Transfer of Fluid with and without Heat Source in Channels with Constant Wall Temperature. NACA TN, 2863. 1952

2. Hossain, M. A. and Takhar, H. S. Radiation Effects on Mixed Convection Along a Vertical Plate with Uniform Surface Temperature. Heat and Mass Transfer, 1996, 31, 243-248. https://doi.org/10.1007/BF02328616

3. Magyari E. and Keller B., Heat and mass transfer in the boundary layers on an exponentially stretching continuous surface, J. Phys. Appl. Phys. 32,1999, $577-585$.

4. Chen, C. H. Heat and Mass Transfer in MHD Flow by Natural Convection from a Permeable, inclined Surface with Variable Wall Temperature and Concentration. 2004,Acta Mechanica, 172, 219-235.

5. Anjali Devi, S. P. and Ganga, B. Viscous Dissipation Effects on Non-Linear MHD Flow in a Porous Medium over a Stretching Surface,2009, International Journal of Applied Mathematics and Mechanics, 5, 45-59.

6. Ishak A., MHD boundary layer flow due to an exponentially stretching sheet with radiation effect, 2011, SainsMalaysiana, 40, $391-5$.

7. Ferdows, M., Nazmul and Ota, M. Thermophoresis and Chemical Reaction Effects on MHD Natural Convective Heat and Mass Transfer Flow in a Rotating Fluid Considering Heat and Mass Fluxes, 2011, Canadian Journal on Science and Engineering Mathematics, 2, 114-139.

8. Pavithra G. M. and Gireesha B. J., Effect of Internal Heat Generation / Absorption on Dusty Fluid Flow over an Exponentially Stretching Sheet with Viscous Dissipation, 2013, Journal of Mathematics, http://dx. DOI. Org/ 10. $1155 / 2013 / 2013 / 583615$

9. Nadeem S., Rashid Mehmood and NorenSher Akbar, Optimized analytical solution for the oblique flow of a Casson-nano fluid 
with convective boundary conditions" Internal Journal of Thermal Science, 78,2014, 90 - 100.

10. Mukhopadhyay S., Iswar Chandra Mondal, and Hayat T., MHD boundary layer flow of a Casson fluid passing through an exponentially stretching porous surface with thermal radiation,,2014Chinese Phys. B, 23(10), 104701.

11. Shateyi, S. and Marewo, G.T. (2014) Numerical Analysis of MHD Stagnation Point Flow of Casson Fluid, Heat and Mass Transfer over a Stretching Sheet. Proceedings of the 7th International Conference on Finite Differences, Finite Elements, Finite Volumes, Boundary Elements, Gdansk, 15-17 May 2014, 128-132

12. Mahanta G. and Shaw S., 3D Casson fluid flow past a porous linearly stretching sheet with convective boundary condition, 2015 Alexandria Engineering Journal, 54, 653 - 659.

13. N Saidulu and A Venkata Lakshmi, slip effects on MHD flow of Casson fluid over an exponentially stretching sheet in the presence of thermal radiation, heat source/sink and chemical reaction,2016, European Journal of Advances in Engineering andTechnology, 3(1): 47-55

14. AurangZaib,1 KrishnenduBhattacharyya, Dual Solutions of Non-Newtonian Casson Fluid Flow and Heat Transfer over an Exponentially Permeable Shrinking Sheet with Viscous Dissipation, Hindawi Publishing Corporation Modelling and Simulation in Engineering Volume 2016, Article ID 6968371, 8 page

15. Pushpalatha K, Sugunamma V, Reddy JVR, Sandeep N. Heat and Mass transfer in unsteady MHD Casson fluid flow with convective boundary conditions. 2016, Int. J. of Advanced Science and Technology 91: 19-38.

16. M. EswaraRao and S. Sreenadh, MHD boundary layer flow of Casson fluid over a stretching/shrinking sheet through a porous medium,2017, International Journal of Trend in Research and Development, Volume 4(1), ISSN: 2394-9333

17. Pramanik, S. Casson Fluid Flow and Heat Transfer Past an Exponentially Porous Stretching Surface in the Presence of Thermal Radiation,2014, Ain Shams Engineering Journal, 5, 205-212. http://dx.doi.org/10.1016/j.asej.2013.05.003

18. Raju RS, Reddy BM, Reddy GJ. Influence of angle of inclination on unsteady MHD Casson fluid flow past a vertical surface filled by the porous medium in the presence of constant heat flux, chemical reaction and viscous dissipation,2017, of Nano Fluids 6(4): 668-679.

19. Hasan E, Karim, A. Samad, MHD Free Convection Flow past an Inclined Stretching Sheet with Considering Viscous Dissipation and Radiation, 2017,7,152-168

20. Mr. M. S. Hossain, Mr. M. A. Samand \& Mr. M. Mohebujjaman, "Heat And Mass Transfer Of An Mhd Forced Convection Flow Along A Stretching Sheet With Chemical Reaction, Radiation And Heat Generation In Presence Of Magnetic Field”, International Journal of Physics and Research, Vol. 1, No. 1, pp. 30-58

21. Hemant Poonia \& Umashanker, "Radiation Effect on Natural Convection Flow Past an Impulsively Started Infinite Vertical Plate through Porous Medium in the Presence of Magnetic Field and First Order Chemical Reaction”, International Journal of Applied Mathematics \& Statistical Sciences(IJAMSS), Vol. 5, No. 6, pp. 17-28

22. N. Senapati \& R. K. Dhal, "Magnetic Effects of Mass and Heat Transfer on Free Convection Flow through Porous Medium Past an Infinite Vertical Plate in Slip - Flow Regime in the Presence of Chemical Reaction, Variable Suction and Periodic Temperature and Mass Concentration", IMPACT: International Journal of Research in Humanities, Arts and Literature(IMPACT : IJRHAL), Vol. 2, No. 1, pp. 19-28

23. Pradip R. Bodade, Nishant Jogi, Mahesh Gorde, Ravi Paropte \& Ranjan Waghchore, "Use of Internal Threads of Different Pitches to Enhance Heat Transfer in a Circular Channel”, BEST: International Journal of Management, Information Technology and Engineering(BEST : IJMITE), Vol. 2, No. 1, pp. 1-4 